\title{
Optimization example of industrial sewing machines mechanisms
}

\author{
Vlastimil Votrubec Pavel Šidlof \\ VÚTS, a.s. \\ U Jezu 4, 46119 Liberec 4, Czech Republic \\ vlastimil.votrubec@vuts.cz,pavel.sidlof@vuts.cz
}

\begin{abstract}
This paper deals with the modeling and optimization of mechanical system, focusing on industrial sewing machines in order to reduce the vibrations by balancing. It presents creation of model of mechanisms and their optimization using Modelica language through software MathModelica and Mathematica. Both advantages and drawbacks of this approach are described and an example of optimization solution is shown.
\end{abstract}

Keywords: optimization; mechanisms; sewing machine

\section{Introduction}

Our research institution partakes in development of industrial sewing machines focused on reduction of vibrations and noise. These properties significantly influence customer's opinions and lead in this business helps with competition. It is necessary to solve similar problems for other types of machines also.

Vibrations and noise are mainly caused by dynamic forces generated during the movement of mechanisms. To reduce these forces, which grow during the permanent rising of machines performance, different balancing methods are used. The simplest method is balancing by rotatory balancers. More significant reduction of inertial forces is possible to achieve using balancing mechanisms. In our institute industrial used patent was developed in this field.

It is necessary to use appropriate computational software for balance suggestion and optimization. One of the best and universal software is software Mathematica. Industrial sewing machines usually contain complicated system of mechanisms. Their dynamic calculation leads to a large number of equations and parameters. We found that programming of the calculation is difficult and we could easily make mistakes, which were tedious to search. Hence the idea of using software MathModelica to create computational system occurred. Then we can execute optimization and some other calculations in software Mathematica.

\section{Balancing of sewing machines}

Sewing machines contain number of mechanisms that cause generation of inertial forces and vibrations. Main of them is mechanism for needle bar motion and thread feed mechanism. Both are placed in the head of the machine. There are crank mechanism that ensures reversible rectilinear movement of needle bar and four bar mechanism for thread feeder motion. Both mechanisms are driven by upper shaft that is connected to lower shaft by transmission belt. Balancing of these two mechanisms could be done by several methods.

\subsection{Balancing methods}

Common balancing methods of inertial forces balancing use rotatory balancers mounted on crank shaft. This method usually balances only centrifugal forces or transfers first harmonic component of inertial force of needle bar from the direction of needle bar axes to the orthogonal direction. Also this method can't balance higher orders harmonic component of inertial forces, especially in crank mechanism for needle bar motion.

Further, the vibrations are often reduced by increasing of mass and thus increasing of stiffness of sewing machine's head. Mounting in silent-blocks also helps.

Using of balance mechanisms hasn't spread yet, because most of these patented methods are complicated, expensive, require big space inside the machine or they don't balance inertial forces sufficiently. The VÚTS patent, which doesn't have many of these drawbacks, was recently used to balance crank mechanism and partially thread feed mechanism. 


\subsection{Calculation of mechanisms balancing and its limits}

For optimization calculation it is necessary to use convenient software. In these cases software Mathematica was used. During the optimization it is necessary to solve system of equations which includes plenty of parameters. Program written in this way is large and it is easy to make mistakes that are difficult to be found.

Therefore we decided to work with software MathModelica that uses Modelica language and that is compatible with Mathematica. The idea was to create model of mechanisms in model editor using Modelica libraries, especially MultiBody library and then analyze it in Mathematica.

\section{Optimization using Modelica lan- guage and Mathematica}

Modeling of mechanisms has specific requests and many of them aren't implemented in current libraries. Big advantage of this software is the ability to edit elements or even create new element and library.

\subsection{Elements editing and creation}

Most of the problems that we encounter when working with mechanical systems first require detection of kinematic quantities courses at specific points in bodies and inertial forces (torques). However, elements of the MultiBody library have these quantities defined differently and in smaller number than we need. Some kinematic quantities (velocity and acceleration) are calculated resolved to individual body coordinate system, the total inertial force (torque) of the body isn't defined at all and all variables are primarily calculated only for points of the body where the connection to other elements is modeled. It was necessary to remove these drawbacks so a new element of a rigid body (RigidBody) featuring a link of a mechanism was formed.

In the editor of the new element, new quantities and parameters were defined and relevant equations were set up. Kinematic quantities in points of connection to other elements, which we need to know resolved to global coordinate system, were simply multiplied with appropriate transformation matrix. It is also necessary to work with the courses of quantities of an arbitrary point of the body (such as center of mass) which standard element doesn't provide.

Therefore a radius vector of a general point resolved to coordinate system of the body was set up as a new parameter. Other quantities are then calcu- lated from this parameter. The position of this point resolved to global coordinate system is calculated as

$$
r_{G A}=r_{G L}+S \cdot r_{L A},
$$

where $r_{G L}$ is position vector of the body coordinate system origin, $S$ is transformation matrix, $r_{L A}$ is position vector of a point $A$ resolved to the coordinate system of the body and $r_{G A}$ is searched position of a point $A$ resolved to the global coordinate system.

The velocity and acceleration could be calculated as time derivatives of this formula, but function derivative can't be used, because inserting of derivatives isn't compatible with inner logic of the software and calculation is interrupted. For this reason velocity and acceleration of given point is calculated as

$$
\begin{aligned}
& v_{G A}=S \cdot v_{G L}+w \times\left(r_{G A}-r_{L A}\right), \\
& a_{G A}=S \cdot a_{G L}+z \times\left(r_{G A}-r_{L A}\right)+ \\
& +w \times\left(w \times\left(r_{G A}-r_{L A}\right)\right)
\end{aligned}
$$

where $w$ and $z$ are angular velocity and acceleration of the body, indexes $G L$ correspond to the origin of the coordinate system of the body and indexes $G A$ correspond to the searched global velocity and acceleration of the given point.

Inertial force $F$ is defined by multiple of body mass $m$ and its acceleration in the center of mass $a_{G T}$. The acceleration is calculated according to relations $(1,2,3)$, only instead of position vector $r_{L A}$, the position vector of center of mass $r_{L T}$ is inserted

$$
F=m \cdot a_{G T} .
$$

In the similar way inertial torque is calculated, only instead of body mass, moment of inertia resolved to the center of mass and angular acceleration of the body are inserted. This method allows us to

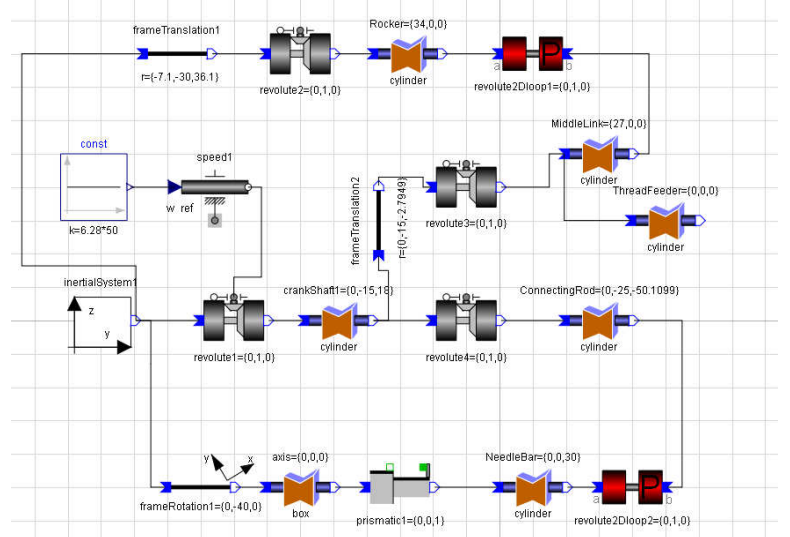

Figure 1: Model of upper mechanism of a sewing machine. 
enlarge elements with new parameters and quantities according as necessary.

There can be relatively a lot of mechanisms inside the sewing machine (according to the type of machine). These mechanisms can form a long chain. Already the model of simple mechanism such as crank mechanism or four bar mechanism from fig. 1 includes about ten elements. For easier work and better orientation it is convenient to assemble these simple mechanisms into blocks and form independent elements from them, which are then connected.

The upper mechanism model of a sewing machine from fig. 1 is then simplified to model that has only five elements (fig. 2). Used elements represent global coordinate system, planar crank mechanism and binary revolute pair replacing four bar mechanism of thread feeder, all for suggested constant value of crank shaft angular velocity which is connected to the CrankMech2D element.

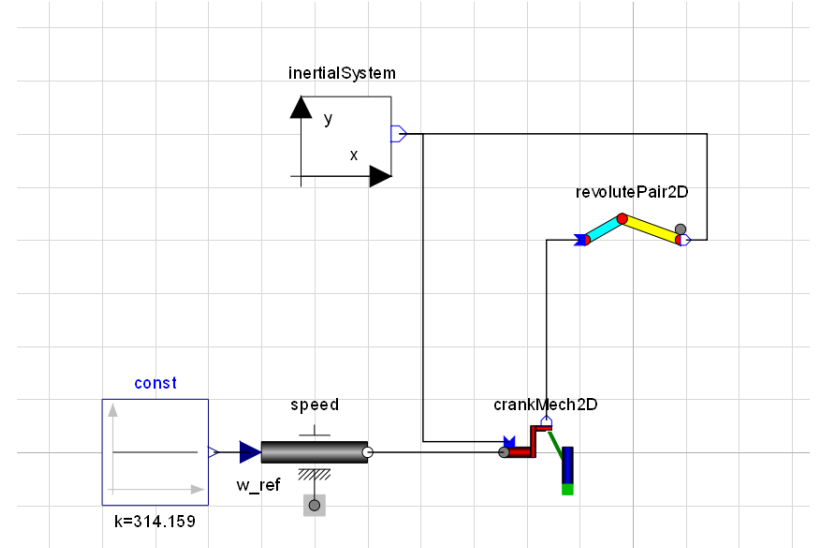

Figure 2: Model of upper mechanisms of sewing machine. Both mechanisms are replaced with single element.

\subsection{Optimization}

Optimization of chosen parameters of the model is then solved in Mathematica. There is created function for finding local minimum that searches the minimal value for every parameters setting. During every step of finding minimum is solved whole system of equations in MathModelica Simulation Center. There is also possible to use any of other operators that the software offers, such as finding local maximum, difference between two courses, finding of roots of derivatives etc.

Generally the optimization is difficult calculation. During the balancing of mechanisms and machines (reduction of total dynamic forces and torques) it is possible to use specific properties of this problem. Usually mechanisms with periodic movement are solved and balanced so that optimal dimensions and moments of inertia of several balancing masses are found. Each body is possible to replace by proper chosen point masses if the flexibility is omitted ( 3 points are enough for plane case). Total inertial force is then sum of inertial forces in these points.

For example it is searching minimum of absolute value of $F$ in vector function

$$
F=m_{1} \cdot a_{1}+m_{2} \cdot a_{2}+\ldots+m_{n} \cdot a_{n} .
$$

If points are chosen conveniently, then only the mass $m_{i}$ of points is necessary to find, not their optimum position. Courses of acceleration $a_{i}$, which are generally given by very complicated relations, are therefore in particular points stable, they can be calculated before the optimization and don't have to be executed in each optimization step. In order to effective calculation using MathModelica, it is necessary to do some modifications and completion in this software.

\subsection{Drawbacks of this approach}

There are several difficulties which make optimization complicated. Firstly it is absence of some mechanical elements and joints in MultiBody library. For instance a lot of mechanisms in sewing and other machines consist of cams and other shape bodies which can't be modeled by means of standard elements. Hence, joint between cam and lift and other elements would help much in mechanisms modeling.

Next problem is time of optimization. Elements of mechanism model form system of equations which includes thousands equations. Already simple mechanism such as four bar mechanism has about 3000 equations, 2500 of them are trivial equations. Although the simulation takes a few seconds, it is executed in every step during the optimization. Finding local minimum is a long process with dozens up to hundreds of steps. This finally causes long time of a calculation depends on model difficulty and number of parameters. Each other used parameter for optimization significantly lengthens the time of calculation.

\subsection{Possible approaches of next process}

One possible solution of the problem with time of optimization was found in solving of whole system of equations independently on Simulation Center which could be much more efficient. MathModelica allows transferring the system of equations to software Mathematica. Mathematica offers many operators for solving equations, the most appropriate operator for this case is function NDSolve that can numerically solve system of differential equations. 
This approach also has some problems which don't enable to sufficiently solve equations for this time.

\subsection{Optimization example}

It will be presented optimization example of balancing on upper mechanisms of sewing machine in this item. As was said before, there are two mechanisms driven by upper shaft, crank mechanism and four bar mechanism. Total inertial force is a sum of particular forces of the bodies. The biggest influences on total inertial force have inertial forces generated by movement of crank shaft and needle bar. The courses of unbalanced total inertial force and its components are on the fig. 3, whereas the crank shaft is rotating 3000 RPM counter clockwise.

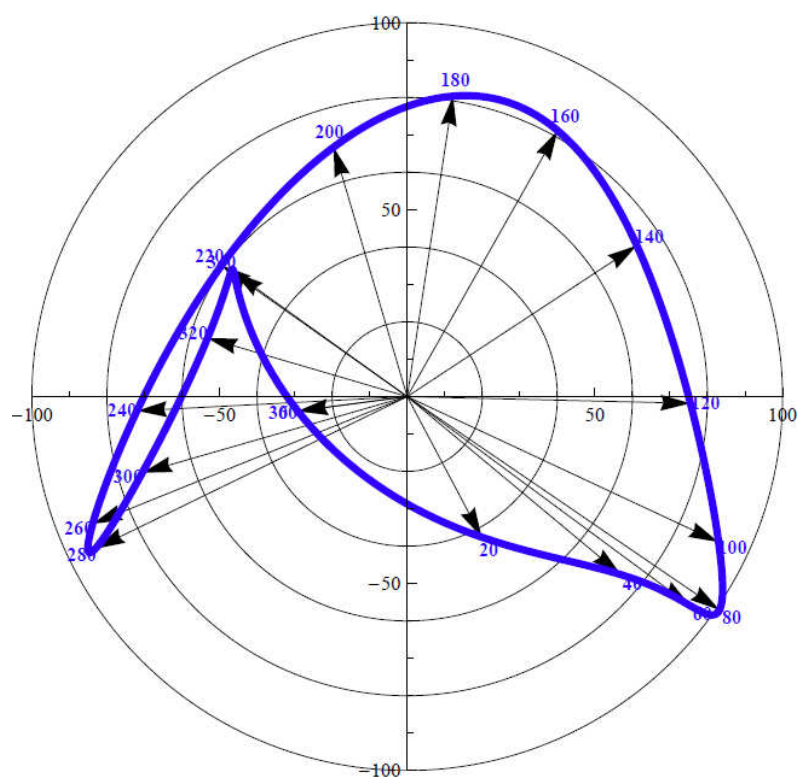

Figure 3: Polar plot of total inertial force of unbalanced upper mechanism. The maximum force is $100,9 N$.

The polar diagram shows resulting force vector. The arrows present vectors for particular drive shaft angle rotation in degrees.

Generally the reduction of total inertial force can be achieved by using two rotatory balancers on the crank shaft. But in this case the crank shaft is prebalanced. Position of center of mass is near the axis of rotation so balancing using rotatory balancers can't help much. Significant reduction of the total force is possible to achieve using a balancing mechanism and one rotatory balancer. In our case it consists from connecting rod that is mounted on the crank shaft on the opposite side of the needle bar crank and balancer (fig. 4). The balancer has small stroke but large mass. Also there is one rotatory balancer placed on the crank shaft.

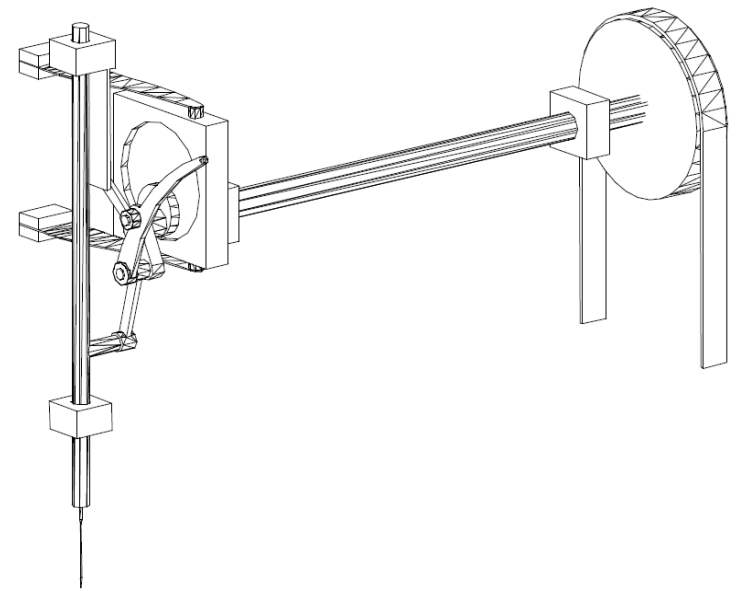

Figure 4: Schema of upper mechanisms of sewing machine. Needle bar mechanism and thread feeder mechanism with additional balancing mechanism.

The dimensions of balancer and balancing mechanism are fixed, so there are two values to optimize, mass of the rotatory balancer and mass of balancer of balancing mechanism. Then in Mathematica is constructed function for calculation of total inertial force, finding the maximum value of that force and the parameters are set. Optimization then implies that the operator for finding local minimum is changing parameters (mass of balancers) until it finds the minimum value.

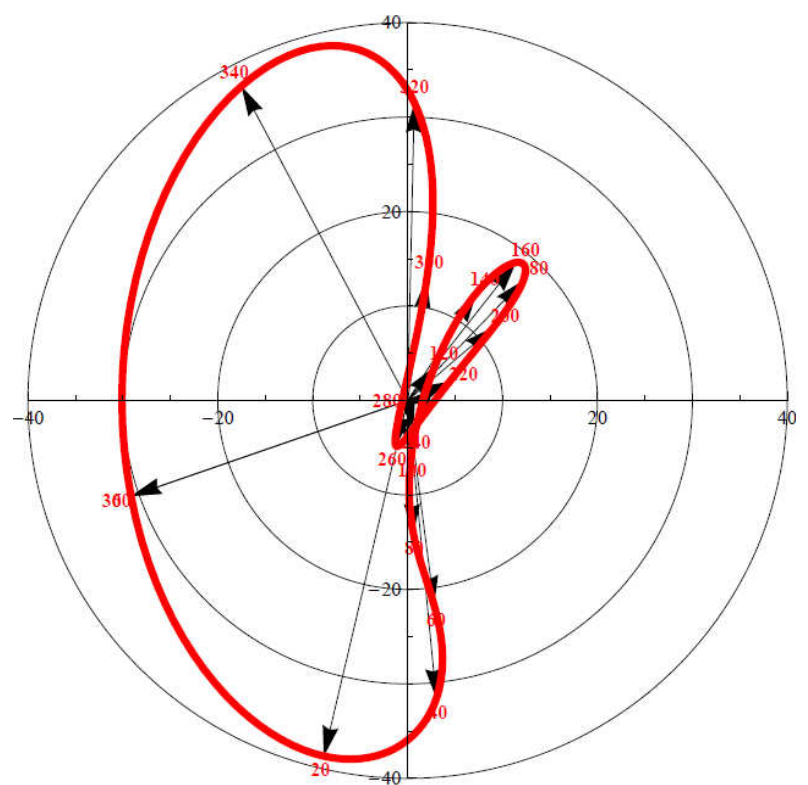

Figure 5: Polar plot of total inertial force of upper mechanisms after the optimization. The maximum force is $38,7 \mathrm{~N}$.

Calculation lasts about 60 minutes $(3 \mathrm{GHz}$ processor, 1.99 GB RAM), for one parameter it lasts 30 minutes. It is clear that for more complicated model 
and more parameters the optimization would take hours. The results are shown on figure 5 . The reduction of total inertial force is relatively significant, maximum of total force descended about $60 \%$.

\section{Conclusion}

Mentioned approach allows modeling of system of mechanisms, executing kinematic and dynamic analysis and optimizing parameters to reduce inertial forces and vibrations. This approach is clear and it reduced mistakes in comparison with other software and methods. On the optimization example of balancers is shown how it is possible to achieve fast and precise results.

On the other hand there is still large area for improving current methods and means in optimizations. For example mechanical elements that corresponds to real links of mechanisms and design problems, possibility to enter more mass parameters etc. 